\title{
The Effectiveness of the Low-Level Laser, Antibiotic and Surgical Therapy in the Treatment of Medication-Related Osteonecrosis of the Jaws: A Case Report
}

\author{
Maria Del Pilar Rodríguez-Sánchez' ${ }^{1}$ Cristian Statkievicz², João Martins de Mello-Neto², Luan Felipe Toro ${ }^{3}$, \\ Ana Paula Farnezzi Bassi ${ }^{2}$, Valdir Gouveia Garcia ${ }^{2}$, Letícia Helena Theodoro ${ }^{4}$, Edilson Ervolino ${ }^{5 *}$ \\ ${ }^{1}$ Montería Dental School Univ. del Sinú - UNISINU, Estrada 1w, 38-153, CEP: 4536534, Montería-COR, Colombia \\ ${ }^{2}$ São Paulo State University (UNESP), School of Dentistry, Department of Surgery and Integrated Clinic, R. José Bonifácio, \\ 1193, CEP: 16015-050, Araçatuba-SP, Brazil \\ ${ }^{3}$ São Paulo State University (UNESP), Institute of Biosciences, R. Professor Dr. Antônio Celso Wagner Zanin, 250, CEP: \\ 18618-689, Botucatu-SP, Brazil \\ ${ }^{4}$ São Paulo State University (UNESP), School of Dentistry, Department of Surgery and Integrated Clinic, R. José Bonifácio, \\ 1193, CEP: 16015-050, Araçatuba-SP, Brazil \\ ${ }^{5}$ São Paulo State University (UNESP), School of Dentistry, Department of Basic Sciences, R. José Bonifácio, 1193, CEP: \\ 16015-050, Araçatuba-SP, Brazil. São Paulo State University (UNESP), Institute of Biosciences, R. Professor Dr. Antônio \\ Celso Wagner Zanin, 250, CEP: 18618-689, Botucatu-SP, Brazil
}

\section{*Correspondence to Edilson Ervolino, DDS, MSc $\mathrm{PhD}$; \\ Phone: +55 18 3636-2801 Email: e.ervolino@unesp.br}

Published online January 18 , 2020

\begin{abstract}
Introduction: Bisphosphonates consist of a range of drugs used in the treatment of osteopathy or some osteotropic malignancies. Medication-related osteonecrosis of the jaw (MRONJ) is a serious adverse consequence of their use. Conventional treatment is not always effective, so it is necessary to resort to the use of adjuvant therapies. This study aimed to evaluate the effectiveness of the association of surgery, antibiotic therapy and low-level laser (LLL) therapy as a treatment strategy for MRONJ through the presentation of a clinical case.

Case Presentation: A 49-year-old female patient presented for the diagnosis and treatment of bone lesions in the maxillae. The patient reported that she had used zoledronate for 1 year. Five years later there were 2 painless bone lesions on both sides of the maxilla, following the extraction of the first upper molars. Clinical, tomographic and histopathologic examination established the diagnosis of MRONJ. The treatment consisted of the curettage of the necrotic bone, antibiotic and thirteen LLL therapy sessions. Integral mucosal healing was observed after a two-month follow-up and no symptoms were detected. The patient was evaluated at 6 and 12 postoperative months without complications.

Conclusion: The combination of surgery, antibiotic therapy, and LLL therapy has shown to be effective in the treatment of MRONJ.

Keywords: Anti-bacterial agents; Diphosphonates; Laser therapy; Oral surgical procedures; Osteonecrosis.
\end{abstract}

\section{Introduction}

A serious adverse consequence of using some oral or intravenous antiresorptive and/or antiangiogenic agents is the medication-related osteonecrosis of the jaw (MRONJ). ${ }^{1}$ This condition is characterized by the presence of the exposed bone or the bone probed through an extraoral or intraoral fistula in the maxillofacial region for a period longer than 8 weeks in the patients who are submitted to previous or current medication with antiresorptive or antiangiogenic agents and do not have any previous history of radiotherapy or metastatic disease within the jaws. ${ }^{1}$
The American Association of Oral and Maxillofacial Surgeons (AAOMS) has classified MRONJ into three stages: I. The exposed necrotic bone with no signs of infection and pain (asymptomatic); II. The exposed necrotic bone with the signs of infection and pain (symptomatic); and III. The exposed, symptomatic and infected bone, associated with the presence of fistulas, maxillary sinus and/or pathological fractures. ${ }^{1}$ Bisphosphonates (BPs) are among the primary agents that result in the occurrence of MRONJ. BPs are used within the current pharmacological arsenal against osteoclastmediated bone loss and for the treatment of some

Please cite this article as follows: Del Pilar Rodríguez-Sánchez M, Statkievicz C, de Mello-Neto JM, Toro LF, Bassi APF, Garcia VG, et al. The effectiveness of the low-level laser, antibiotic and surgical therapy in the treatment of medication-related osteonecrosis of the jaws: a case report. J Lasers Med Sci. 2020;11(1):98-103. doi:10.15171/jlms.2020.16. 
osteoporotic conditions, hypercalcemia, Paget's disease, and multiple myeloma and the control of malignancies. ${ }^{2}$ $\mathrm{Marx}^{3}$ were the first to relate the use of BPs with the onset of MRONJ. MRONJ is less common in patients who use BPs orally; however, in patients who use long-term BPs, as zoledronate, via parenteral, prescribed for adjuvant oncologic therapy, MRONJ can affect $1 \%$ to $12 \%$ of patients. ${ }^{4}$ Since BPs have cumulative effect on mineralized tissues, this incidence may increase up to $21 \%$ after the third year of continuing therapy. ${ }^{4}$

Although MRONJ has been described for more than a decade, its pathogenesis is still poorly understood, which hinders its prevention and treatment. In addition, there are not any established and definitive clinical protocols to be followed in the treatment of MRONJ. The currently proposed treatments are based on the clinical staging of the disease, ranging from mouthwash with chlorhexidine and antibiotics to debridement and surgical resection of the affected area. ${ }^{1}$ However, this therapeutic model has proven inadequate, and in some cases, recurrences are common, which shows that there is a tremendous need to involve other adjuvant therapies.

In vitro and in vivo studies have shown that BPs have negative effects on several cell lines and consequently compromise the ability of the tissue to perform repair..$^{5-7}$ Thus, the use of adjuvant therapies with biostimulatory effects could promote benefits, including the cases in which the standard protocol does not achieve satisfactory results.

In this context, some studies have used platelet-rich plasma (PRP), ${ }^{8}$ hyperbaric oxygen, ${ }^{9}$ bone morphogenetic protein ${ }^{10}$ and low-level laser (LLL) as adjuvant therapies for the treatment of MRONJ.1112 Interestingly, LLL therapy has shown promising results for the treatment of this condition. ${ }^{11-13}$

The basic mechanism underlying LLL therapy has not completely been elucidated yet. The primary reactions occur at the mitochondrial level, specifically in the electron transport chain, where irradiation promotes photoreceptors activation, which results in increased energy potential cells. Other reactions increase cell proliferation and cell differentiation when they occur, involving the transduction of signals to the cytoplasm level. ${ }^{14}$

Previous animal studies by our research group ${ }^{15}$ and other research groups ${ }^{16-18}$ have shown that LLL therapy after extraction improves the alveolar repair process. Similarly, clinical studies have also indicated that LLL therapy has positive effects on the main signs and symptoms during the post-extraction wound healing process. ${ }^{12,19-21}$

In some clinical studies, Vescovi et al, ${ }^{12,22}$ showed a higher therapeutic success rate when LLL therapy was associated with conventional therapy in the treatment of MRONJ. Corroborating these findings, other literature reviews also report the benefits of the inclusion of LLL as a promising option for MRONJ management. ${ }^{23,24}$ Therefore, this study aimed to evaluate the effect of the association of surgery intervention, antibiotic therapy, and LLL therapy, as a treatment strategy for MRONJ through the presentation of a case.

\section{Case Presentation}

A 49-year-old female patient, who was a smoker, presented at the clinical division of School of Dentistry, Araçatuba, São Paulo State University (UNESP), Brazil for the diagnosis and treatment of 2 bone lesions located in the bilateral molar region of the maxilla. Initially, ethical considerations were given and written informed consent was obtained from the patient to be submitted to care and subsequent treatments. During anamnesis, the patient reported the use of zoledronate (zoledronic acid), $4 \mathrm{mg} / \mathrm{month}$ for 12 months for the treatment of an osteoblastoma in the ribs. Five years after stopping the use of this drug, the patient underwent the extraction of the first upper molars; however, after 6 months, the dental sockets showed no clinical signs of tissue repair. The patient reported the use of $1500 \mathrm{mg} / \mathrm{d}$ of amoxicillin for 3 months. At the end of this period, as was found during a physical examination, an exposed bone was noted with painful symptoms and drainage of the purulent collection in the posterior region of both sides of the maxilla (Figure $1 \mathrm{~A}$ and $1 \mathrm{~B})$. Radiographic examination and a computed tomography (CT) scan of the patient exhibited, on both sides, an irregular lesion with ill-defined margins, radiolucent aspect, and alveolar bone resorption with maxillary sinus involvement (Figure 1C, 1D and 1E).

Based on the history, physical examination, radiography, and tomography, the diagnosis of MRONJ (stage III) was established and confirmed with a posterior histopathologic exam. The treatment consisted of surgical debridement therapy, antibiotic administration, and multiple LLL therapy sessions.

\section{Surgical Procedures}

The patient was submitted to all surgical procedures under local anesthesia. The detachment of the mucosa was held in the wound edges. The necrotic bone was removed by using a Dietrich forceps (Figure $2 \mathrm{~A}$ and $2 \mathrm{~B}$ ). Then, the curettage of the necrotic bone was conducted until the bone walls were bleeding (Figure 2C). Bone defects were found to involve the maxillary sinus which was confirmed by the use of the Valsalva maneuver. A classic ball-shaped bur was used to perform the osteoplasty and to remove the sharp angles of the bone. Wounds were abundantly irrigated with a sterile saline physiologic solution and the first LLL therapy session was performed during the transoperative period (Figure 2D).

LLL therapy was performed with the use of a GaAlAs (gallium-aluminum arsenium) diode laser (Thera Lase ${ }^{\circledR}$, D.M.C. Ltd, São Carlos, SP, Brazil) with a wavelength of $808 \mathrm{~nm}$ and a spot size of $0.0283 \mathrm{~cm}^{2}$. LLL was performed 

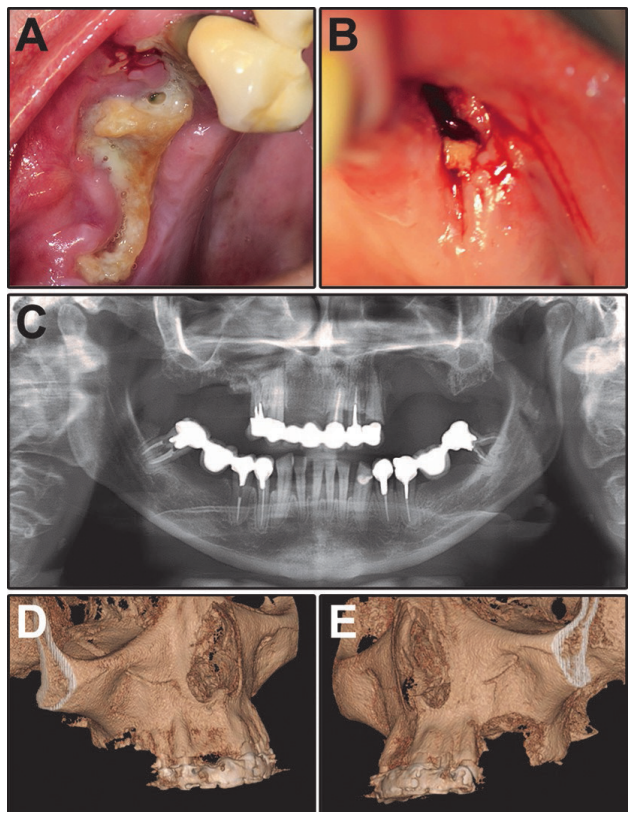

Figure 1. Clinical and Imaging Characteristics of the Lesion in the Preoperative Period. (A-B) Clinical aspects of the right (A) and left (B) maxillary lesions. (C-E) Radiographic (C) and tomographic (D-E) appearance.

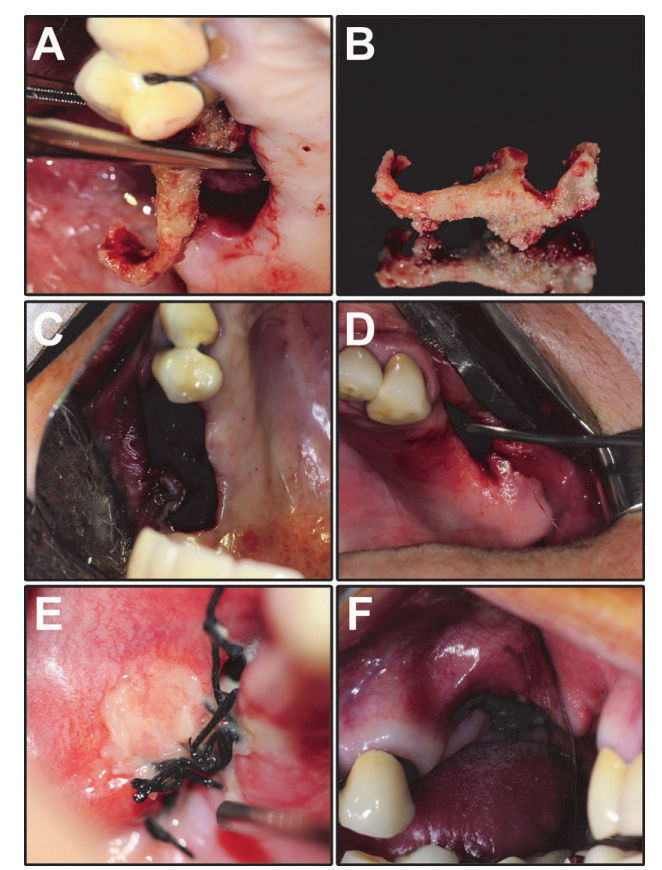

Figure 2. Surgical Transoperative. (A) shows the removal of the bone sequestration, which is evidenced in (B). In (C), the lesion located in the maxilla can be observed at the end of the surgery. (D) shows an injury located in the maxilla soon after the surgery had been completed and a laser tip positioned at a point of irradiation. (E) and (F) show the aspect of the surgical sites after the suture.

with an energy density of $19.43 \mathrm{~J} / \mathrm{cm}^{2} /$ point, $4.4 \mathrm{~J}$ of total energy, a power of $50 \mathrm{~mW}$, a power density of $1.76 \mathrm{~W} /$ $\mathrm{cm}^{2}$, and in a continuous mode. The laser was applied to 6 points on the wound edges and to 2 points in the center (Figure 2D) for 11 seconds for each dot, totaling 88 seconds on each side and a cumulative dose of 155.44 $\mathrm{J} / \mathrm{cm}^{2}$. The first LLL therapy session was performed even before making the suture. After this, the sessions of LLL therapy were performed twice a week in the first month, followed by an LLL therapy session once a week in the second month. All necessary biosafety measures were employed during LLL therapy, including the disinfection of the laser tip and the use of protection eyeglasses by the patient and the operator.

A flap in the mucosa was performed and sutured in order to cover the defect and allow first intention healing. (Figure $2 \mathrm{E}$ and $2 \mathrm{~F}$ ). Antibiotic therapy with clindamycin $(1200 \mathrm{mg} /$ day $)$ was administered for 14 days following the procedure.

Part of the necrotic bone was sent to the Pathology Laboratory of School of Dentistry, Araçatuba - UNESP for histopathologic examination. The histopathological analysis showed an extensive area of necrotic bone tissue characterized by empty gaps of o steocytes and circumscribed by intense chronic inflammatory infiltrate and necrotic debris with microbial colonies of bacteria, which histopathologically proved the clinical hypothesis of MRONJ (Figure 3A, 3B and 3C).

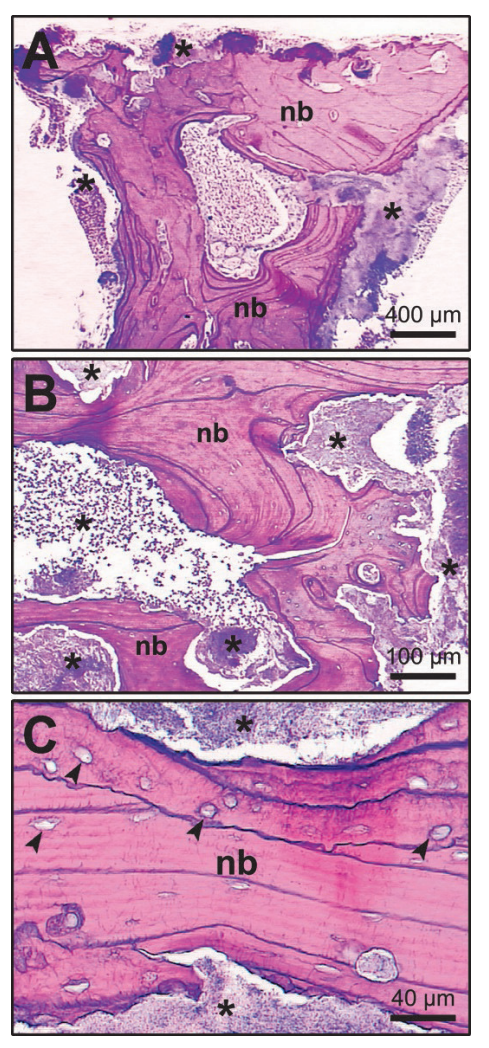

Figure 3. Histopathological Characteristics of the Lesion. (A - C) Photomicrographs evidencing the histological aspect of the bone sequestration. Note the non-vital bone tissue and marrow spaces filled with necrotic cell remnants, inflammatory infiltrate and bacterial colonies. Abbreviations and symbols: asterisks, necrotic remains, and bacterial colonies; Arrows, empty gaps; nb, necrotic bone. Scale bar: $400 \mu \mathrm{m}(\mathrm{A})$, $100 \mu \mathrm{m}(B), 40 \mu \mathrm{m}(\mathrm{C})$. Staining: Hematoxylin and Eosin. 
During the laser treatment, the patient was monitored weekly and radiographic and tomographic exams were done after 12 postoperative months. Follow-up in the periods of 3, 6 (Figure 4A, 4B and 4C) and 12 postoperative months showed that the lesion area completely recovered with healthy mucosa covering the neoformed bone (Figure 4D and 4E).

\section{Discussion}

MRONJ has become a major concern in dentistry due to the difficulty in obtaining a cure, substantially compromising the life quality of the patients. According to Ruggiero and Woo, ${ }^{25}$ BPs have a half-life of about 10 years. We observed the development of MRONJ 5 years after the completion of treatment with zoledronic acid. In an epidemiological study, Otto et $\mathrm{al}^{26}$ identified the main risk factors for the development of MRONJ. According to this study, the patient had all the risk factors for developing the disease, such as advanced age, female gender, smoker, chronic intravenous use of zoledronate (a nitrogen BPs) and the fact that she had been subjected to an invasive surgery as a tooth extraction. ${ }^{26,27}$ The complication associated with this case is the fact that the patient presents a maxillary sinus involvement, representing MRONJ in stage III. The results from a study by Berrone et $\mathrm{al}^{28}$ showed that the treatment of 5 patients with MRONJ in stage III with surgical debridement, antibiotics and closing of wounds using the buccal fat pad for bone coverage was successful. There was no recurrence during a twelve-month follow-up. The authors reported successful treatment, supposedly stimulated by the rich blood supply to the area. ${ }^{28}$ The increased prevalence of MRONJ is in the jaw, in particular in the premolar and molar regions, and it is attributed to the lower blood supply to the bone tissue of this area, which makes the strategies for increasing the vascularization necessary.

Ristow and Pauke $^{29}$ defined as successful surgical therapy for MRONJ when all necrotic bone tissue has been removed, there is completely repair and recoating of the overlying mucosa and no symptoms are reported for at least 4 weeks after the surgical procedure. According to AAOMS, a recommended treatment should be the limitation of infection, the relief of pain symptoms, and the control of the necrosis progression. ${ }^{1}$ Antibiotic therapy and a surgical procedure may not be sufficient to cure MRONJ in some cases, ${ }^{30}$ so we choose to associate it with adjuvant therapy. LLL therapy, which is also called photobiomodulation, has properties that could favor the process of tissue repair. ${ }^{15,31}$

LLL therapy is able to modulate the metabolism of human osteoblasts by increasing their proliferation and differentiation. ${ }^{32}$ Moreover, LLL therapy is claimed to have beneficial analgesic and anti-inflammatory effects. ${ }^{33}$ It promotes bone formation and soft tissue healing and has important angiogenic properties for tissue repair. ${ }^{34}$ Positive effects, which have been suggested and confirmed

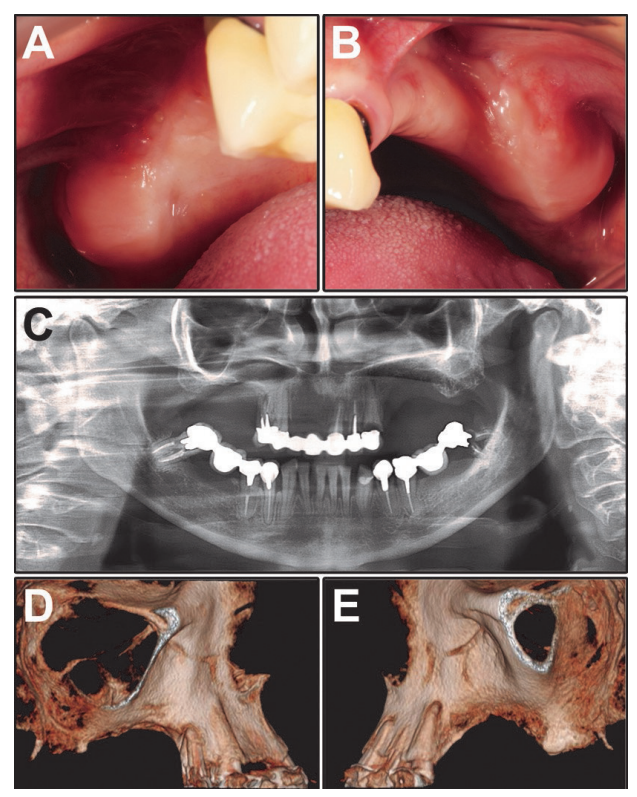

Figure 4. Clinical and Imaging Characteristics of the Lesion Site in the Postoperative Period (1 Year). (A-B) Clinical aspects of the posterior region of the right $(\mathrm{A})$ and left $(\mathrm{B})$ maxillae. (C-E) Radiographic $(\mathrm{C})$ and tomographic (D-E) aspect of both sides of the maxilla.

with a meta-analysis, include an increase in collagen synthesis and tensile strength, accelerating the resolution of the inflammatory process and the diminution of healing time and wound size. ${ }^{35}$

These facts could explain the beneficial effect of LLL therapy when it is associated with the conventional treatment of MRONJ. ${ }^{12,22}$ The laser parameters, including the energy that is delivered, power density, pulse duration, appropriate treatment, timing and repetition, and the appropriate anatomical location must be adequate to create a biostimulatory effect. ${ }^{23}$

In this case report, we used the laser emitting GaAlAs at a wavelength of $808 \mathrm{~nm}$ with an energy density of $19.4 \mathrm{~J} /$ $\mathrm{cm}^{2} /$ point (8 points) for a period of 2 months ( 13 sessions). Other protocols have been described for the treatment of MRONJ. Romeo et $\mathrm{al}^{36}$ used a double diode laser (GaAs) simultaneously emitting at 2 different wavelengths, 650 $\mathrm{nm}$ and $904-910 \mathrm{~nm}$. Vescovi et $\mathrm{al}^{37}$ applied a LLL therapy with previous irrigations with a $10 \%$ povidone iodine solution and an emitter Nd: YAG laser at a wavelength of $1064 \mathrm{~nm}$, power of $2.25 \mathrm{~W}$ and frequency of $15 \mathrm{~Hz}$ in a unfocused mode and $2 \mathrm{~mm}$ away from the tissues for 1 minute (power density, $1562.5 \mathrm{~W} / \mathrm{cm}^{2}$, fluence, $7 \mathrm{~J} / \mathrm{cm}^{2}$ ) 5 times repeatedly. Altay et $\mathrm{al}^{31}$ employed a GaAlAs device with a wavelength of $808 \mathrm{~nm}$, power of $0.5 \mathrm{~W}$, and an energy density of $5 \mathrm{~J} / \mathrm{cm}^{2}$. With regard to the number and frequency of LLL therapy sessions, there was a variation in the studies from one ${ }^{37}$ to 5 sessions ${ }^{31}$ and from $650^{36}$ to $1562.5 \mathrm{~nm}^{37}$ respectively. All of these protocols showed good results, as did this case report by improving tissue repair, ${ }^{31}$ complete mucosal healing in about 2 weeks ${ }^{37}$ and a reduction of pain..$^{36}$ The number of irradiation protocol 
variables in laser use, as well as the different affected areas and their intensity that occur with MRONJ, the different laser devices and wavelengths, and the difficulty in standardizing of the amount of energy used make it difficult to establish treatment protocols for the treatment of this pathological condition. Romeo et $\mathrm{al}^{36}$ encourage an individual protocol for each situation.

It has been recognized that Actinomyces play an important role in MRONJ pathophysiology, although its mechanism has not completely been elucidated yet. ${ }^{1}$ In this case, after extraction, the patient continued the use of $1500 \mathrm{mg}$ of Amoxicillin per day for 3 months, which was not sufficient to prevent the necrosis progression. Martins et $\mathrm{al}^{38}$ associated surgical debridement, antibiotic therapy, LLL therapy to PRP in the treatment of MRONJ, achieving a better result when the combination of therapies was used. Perhaps the key to curing MRONJ was to control bacterial and remove the necrotic tissue, but above all to biostimulate the area, which was achieved by adjuvant therapy with LLL. However, more studies must be conducted to prove the beneficial effects of LLL therapy for the treatment of MRONJ.

\section{Conclusion}

Based on the results obtained from this clinical case report and considering its due limits, we concluded that the combination of surgery, antibiotic therapy, and LLL therapy was effective in the treatment of MRONJ, which is put forth as a promising option for adjunctive use in the treatment of this pathological condition.

\section{Ethical Considerations}

Written informed consent was obtained from the patient to participate in this research and for publication of this case report, including its accompanying data and images.

\section{Conflict of Interests}

The authors declare no conflict of interest.

\section{Funding}

This research did not receive any grants from funding agencies.

\section{References}

1. Ruggiero SL, Dodson TB, Fantasia J, Goodday R, Aghaloo T, Mehrotra B, et al. American association of oral and maxillofacial surgeons position paper on medicationrelated osteonecrosis of the jaws - 2014 update. J Oral Maxillofac Surg. 2014;72(10):1938-56. doi: 10.1016/j. joms.2014.04.031.

2. Drake MT, Clarke BL, Khosla S. Bisphosphonates: mechanism of action and role in clinical practice. Mayo Clin Proc. 2008;83(9):1032-45. doi: 10.4065/83.9.1032.

3. Marx RE. Pamidronate (Aredia) and zoledronate (Zometa) induced avascular necrosis of the jaws: a growing epidemic. J Oral Maxillofac Surg. 2003;61(9):1115-7. doi: 10.1016/ s0278-2391(03)00720-1.
4. Gómez Font R, Martínez García ML, Olmos Martínez JM. Osteochemonecrosis of the jaws due to bisphosphonate treatments. Update. Med Oral Patol Oral Cir Bucal. 2008;13(5):e-318-24.

5. Pansani TN, Basso FG, Turirioni AP, Kurachi C, Hebling J, de Souza Costa CA. Effects of low-level laser therapy on the proliferation and apoptosis of gingival fibroblasts treated with zoledronic acid. Int J Oral Maxillofac Surg. 2014;43(8):1030-4. doi: 10.1016/j.ijom.2014.02.011.

6. Reid IR, Bolland MJ, Grey AB. Is bisphosphonate-associated osteonecrosis of the jaw caused by soft tissue toxicity? Bone. 2007;41(3):318-20. doi: 10.1016/j.bone.2007.04.196.

7. Saito T, Izumi K, Shiomi A, Uenoyama A, Ohnuki H, Kato H, et al. Zoledronic acid impairs re-epithelialization through down-regulation of integrin $\alpha \mathrm{v} \beta 6 \mathrm{a}$ and transforming growth factor beta signalling in a three-dimensional in vitro wound healing model. Int J Oral Maxillofac Surg. 2014;43(3):373-80. doi: 10.1016/j.ijom.2013.06.016.

8. Bocanegra-Perez S, Vicente-Barrero M, Knezevic $\mathrm{M}$, Castellano-Navarro JM, Rodríguez-Bocanegra E, Rodríguez-Millares J, et al. Use of platelet-rich plasma in the treatment of bisphosphonate-related osteonecrosis of the jaw. Int J Oral Maxillofac Surg. 2012;41(4):1410-5. doi: 10.1016/j.ijom.2012.04.020.

9. Freiberger JJ. Utility of hyperbaric oxygen in treatment of bisphosphonate-related osteonecrosis of the jaws. $J$ Oral Maxillofac Surg. 2009;67:96-106. doi: 10.1016/j. joms.2008.12.003.

10. Rahim I, Salt S, Heliotis M. Successful long-term mandibular reconstruction and rehabilitation using nonvascularised autologous bone graft and recombinant human BMP-7 with subsequent endosseous implant in a patient with bisphosphonate-related osteonecrosis of the jaw. Br J Oral Maxillofac Surg. 2015;53(9):870-4. doi: 10.1016/j.bjoms.2015.08.006.

11. Lee JY, Kim IR, Park BS, Kim YD, Chung IK, Song JM, et al. Effect of low-level laser therapy on oral keratinocytes exposed to bisphosphonate. Lasers Med Sci. 2015;30(2):63543. doi: 10.1007/s10103-013-1382-6.

12. Vescovi P, Meleti M, Merigo E, Manfredi M, Fornaini C, Guidotti R, et al. Case series of 589 tooth extractions in patients under bisphosphonates therapy. Proposal of a clinical protocol supported by Nd:YAG low-level laser therapy. Med Oral Patol Oral Cir Bucal. 2013;18(4):680-5. doi:10.4317/medoral.18812.

13. Scoletta M, Arduino PG, Reggio L, Dalmasso P, Mozzati M. Effect of low-level laser irradiation on bisphosphonateinduced osteonecrosis of the jaws: preliminary results of a prospective study. Photomed Laser Surg. 2010;28(2):17984. doi: 10.1089/pho.2009.2501.

14. de Freitas LF, Hamblin MR. Proposed mechanisms of photobiomodulation or low-level light therapy. IEEE J Sel Top Quantum Electron. 2016;22(3):7000417. doi: 10.1109/ JSTQE.2016.2561201.

15. Statkievicz C, Toro LF, de Mello-Neto JM, de Sá DP, Casatti CA, Issa JPM, et al. Photomodulation multiple sessions as a promising preventive therapy for medication-related osteonecrosis of the jaws after tooth extraction in rats. J Photochem Photobiol B. 2018;184:7-17. doi: 10.1016/j. jphotobiol.2018.05.004.

16. Noda M, Aoki A, Mizutani K, Lin T, Komaki M, Shibata S, 
et al. High-frequency pulsed low-level diode laser therapy accelerates wound healing of tooth extraction socket: an in vivo study. Lasers Surg Med. 2016;48(10):955-64. doi: 10.1002/lsm.22560.

17. Park JB, Ahn SJ, Kang YG, Kim EC, Heo JS, Kang KL. Effects of increased low-level diode laser irradiation time on extraction socket healing in rats. Lasers Med Sci. 2015;30(2):719-26. doi: 10.1007/s10103-013-1402-6.

18. Korany NS, Mehanni SS, Hakam HM, El-Maghraby EM. Evaluation of socket healing in irradiated rats after diode laser exposure (histological and morphometric studies). Arch Oral Biol. 2012;57(7):884-91. doi: 10.1016/j. archoralbio.2012.01.009.

19. Eroglu CN, Keskin Tunc S. Effectiveness of single session of low-level laser therapy with a $940 \mathrm{~nm}$ wavelength diode laser on pain, swelling, and trismus after impacted third molar surgery. Photomed Laser Surg. 2016;34(9):406-10. doi: 10.1089/pho.2016.4101.

20. Ferrante M, Petrini M, Trentini P, Perfetti G, Spoto G. Effect of low-level laser therapy after extraction of impacted lower third molars. Lasers Med Sci. 2013;28(3):845-9. doi: 10.1007/s10103-012-1174-4.

21. Batinjan G, Filipović Zore I, Rupić I, Bago Jurič I, Zore Z, Gabrić Pandurić D. Assessing health-related quality of life with antimicrobial photodynamic therapy (APDT) and low level laser therapy (LLLT) after third molar removal. $J$ Lasers Med Sci. 2013;4(3):120-6.

22. Vescovi P, Giovannacci I, Merigo E, Meleti M, Manfredi M, Fornaini C, et al. Tooth extractions in high-risk patients under bisphosphonate therapy and previously affected with osteonecrosis of the jaws: surgical protocol supported by low-level laser therapy. J Craniofac Surg. 2015;26(3):696-9. doi: 10.1097/SCS.0000000000001665.

23. Latifyan S, Genot MT, Klastersky J. Bisphosphonaterelated osteonecrosis of the jaw: a review of the potential efficacy of low-level laser therapy. Support Care Cancer. 2016;24(9):3687-93. doi: 10.1007/s00520-016-3139-9.

24. Weber JB, Camilotti RS, Ponte ME. Efficacy of laser therapy in the management of bisphosphonate-related osteonecrosis of the jaw (BRONJ): a systematic review. Lasers Med Sci. 2016;31(6):1261-72. doi: 10.1007/s10103016-1929-4.

25. Ruggiero SL, Woo SB. Biophosphonate-related osteonecrosis of the jaws. Dent Clin North Am. 2008;52(1):111-28. doi:10.1016/j.cden.2007.09.002.

26. Otto S, Schreyer C, Hafner S, Mast G, Ehrenfeld M, Stürzenbaum S, et al. Bisphosphonate-related osteonecrosis of the jaws - characteristics, risk factors, clinical features, localization and impact on oncological treatment. $J$ Craniomaxillofac Surg. 2012;40(4):303-9. doi: 10.1016/j. jcms.2011.05.003.

27. Saad F, Brown JE, Van Poznak C, Ibrahim T, Stemmer SM, Stopeck AT, et al. Incidence, risk factors, and outcomes of osteonecrosis of the jaws: integrated analysis from tree blinded active-controlled phase III trials in cancer patients with bone metastases. Ann Oncol. 2012;23(5):1341-7. doi: 10.1093/annonc/mdr435.

28. Berrone M, Florindi FU, Carbone V, Aldiano C, Pentenero M. Stage 3 Medication-Related Osteonecrosis of the Posterior Maxilla: Surgical Treatment Using a Pedicled Buccal Fat Pad Flap: Case Reports. J Oral Maxillofac Surg. 2015;73(11):2082-6. doi: 10.1016/j.joms.2015.06.165.

29. Ristow O, Pautke C. Auto-fluorescence of the bone and its use for delineation of bone necrosis. Int J Oral Maxillofac Surg. 2014;43(11):1391-3. doi: 10.1016/j.ijom.2014.07.017.

30. Hoefert S, Eufinger H. Relevance of a prolonged preoperative antibiotic regime in the treatment of bisphosphonaterelated osteonecrosis of the jaw. J Oral Maxillofac Surg. 2011;69(2):362-80. doi: 10.1016/j.joms.2010.06.200.

31. Altay MA, Tasar F, Tosun E, Kan B. Low-level laser therapy supported surgical treatment of bisphosphonate related osteonecrosis of jaws: a retrospective analysis of 11 cases. Photomed Laser Surg. 2014;32(8):468-75. doi: 10.1089/ pho.2014.3742.

32. Oliveira FA, Matos AA, Santesso MR, Tokuhara CK, Leite $\mathrm{AL}$, Bagnato VS, et al. Low intensity lasers differently induce primary human osteoblast proliferation and differentiation. J Photochem Photobiol B. 2016;163:14-21. doi: 10.1016/j.jphotobiol.2016.08.006.

33. Spanemberg JC, Figueiredo MA, Cherubini K, Salum FG. Low-level laser therapy: a review of its applications in the management of oral mucosal disorder. Altern Ther Health Med. 2016;22(6):24-31.

34. de Medeiros ML, Araújo-Filho I, da Silva EM, de Sousa Queiroz WS, Soares CD, de Carvalho MG, et al. Effect of low-level laser therapy on angiogenesis and matrix metalloproteinase-2 immunoexpression in wound repair. Lasers Med Sci. 2017;32:(1):35-43. doi: 10.1007/s10103016-2080-y.

35. Enwemeka CS, Parker JC, Dowdy DS, Harkness EE, Sanford LE, Woodruff LD. The efficacy of low-power lasers in tissue repair and pain control: a meta-analysis study. Photomed Laser Surg. 2004;22(4):323-9. doi: 10.1089/ pho.2004.22.323.

36. Romeo U, Galanakis A, Marias C, Vecchio AD, Tenore G, Palaia G, et al. Observation of pain control in patients with bisphosphonate-induced osteonecrosis using low level laser therapy: preliminary results. Photomed Laser Surg. 2011;29(7):447-52. doi: 10.1089/pho.2010.2835.

37. Vescovi P, Merigo E, Meleti M, Manfredi M, Fornaini C, Nammour S, et al. Conservative surgical management of stage I bisphosphonate-related osteonecrosis of the jaw. Int J Dent. 2014. doi: 10.1155/2014/107690.

38. Martins MA, Martins MD, Lascala CA, Curi MM, Migliorati CA, Tenis CA, et al. Association of laser phototherapy with PRP improves healing of bisphosphonate-related osteonecrosis of the jaws in cancer patients: a preliminary study. Oral Oncol. 2012;48(1):79-84. doi: 10.1016/j. oraloncology.2011.08.010. 